\title{
UAV POSITIONING AND COLLISION AVOIDANCE BASED ON RSS MEASUREMENTS
}

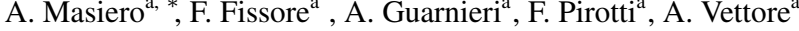 \\ ${ }^{\text {a }}$ Interdepartmental Research Center of Geomatics (CIRGEO), University of Padova, \\ Viale dell'Università 16, Legnaro (PD) 35020, Italy - \\ masiero@ dei.unipd.it \\ (francesca.fissore,alberto.guarnieri, francesco.pirotti, antonio.vettore)@unipd.it
}

KEY WORDS: UAV, Positioning, Collision avoidance, RPAS, Statistical filtering

\begin{abstract}
:
In recent years, Unmanned Aerial Vehicles (UAVs) are attracting more and more attention in both the research and industrial communities: indeed, the possibility to use them in a wide range of remote sensing applications makes them a very flexible and attractive solution in both civil and commercial cases (e.g. precision agriculture, security and control, monitoring of sites, exploration of areas difficult to reach).

Most of the existing UAV positioning systems rely on the use of the GPS signal. Despite this can be a satisfactory solution in open environments where the GPS signal is available, there are several operating conditions of interest where it is unavailable or unreliable (e.g. close to high buildings, or mountains, in indoor environments). Consequently, a different approach has to be adopted in these cases.

This paper considers the use of WiFi measurements in order to obtain position estimations of the device of interest. More specifically, to limit the costs for the devices involved in the positioning operations, an approach based on radio signal strengths (RSS) measurements is considered.

Thanks to the use of a Kalman filter, the proposed approach takes advantage of the temporal dynamic of the device of interest in order to improve the positioning results initially provided by means of maximum likelihood estimations.

The considered UAVs are assumed to be provided with communication devices, which can allow them to communicate with each other in order to improve their cooperation abilities. In particular, the collision avoidance problem is examined in this work.
\end{abstract}

\section{INTRODUCTION}

The use of Unmanned Aerial Vehicles (UAVs) is becoming more and more frequent in both research and industrial applications: indeed, their possibility to be used in a wide range of remote sensing applications makes them a very flexible and attractive solution in both civil and commercial cases (e.g. precision agriculture, security and control, monitoring of sites, exploration of areas difficult to reach (Zhang and Kovacs, 2012, Maza et al., 2011, Casbeer et al., 2006, Guarnieri et al., 2010)).

UAV positioning system is usually based on the use of the GPS signal: despite being a quite general solution to the positioning problem, positioning system based on the GPS signal actually have some issues in certain operating conditions. More specifically, this positioning approach cannot be applied where the GPS signal is not available or not reliable, and for applications where the positioning precision required to the device is higher than that ensured by the GPS positioning in that environment.

Furthermore, when more than one UAV is simultaneously in action, the strategies initially adopted in the literature were based on a centralized approach (Boivin et al., 2008, Wang et al., 2007), where the computational load is concentrated on a single central computational unit. However, this strategy becomes inefficient when dealing with a large number of devices. Despite in most of the applications of interested the number of UAVs is typically quite limited, it is worth to consider a strategy that efficiently scales with the number of devices, e.g. a distributed approach.

The above considerations motivate the search for alternative options in order to substitute or improve the positioning based on the

\footnotetext{
${ }^{*}$ Corresponding author.
}

GPS signal and, if possible, to make the adopted approach computationally efficient even when dealing with a (possibly) large scale network of UAVs.

Recently, (Luo et al., 2013) proposed to consider a positioning approach based on the use of the radio signal strength: (Luo et al., 2013) considered the use of an extended Kalman filter in order to reduce the bad influence of colored noise on the position estimation algorithm. Then, a cooperative strategy was adopted to ensure collision avoidance between UAVs: each UAV computes estimates of the distance from its neighbors by means of RSS measurements, and, if needed, applies a simple procedure in order to avoid collisions. The risk of collision is determined by checking if the distance between the two UAVs is lower then a safety distance, where the safety distance is determined taking into account of the velocities of the UAVs (velocity information is sent between UAVs by means of WiFi communications), of their distance and of the error on the distance measurement achieved by means of RSS measurements. The adopted collision avoidance procedure is designed to avoid collisions between two UAVs, however it does not allow to deal with a generic number of vehicles.

Similarly to (Luo et al., 2013), this paper considers an approach for position estimation based on the use of the radio signal strength and on the cooperation between devices to avoid collisions. However, differently from (Luo et al., 2013), in order to reduce the computational complexity of the approach, the positioning algorithm is based on the use a linear Kalman filter. Nevertheless, maximum likelihood measurements are used to ensure good performance of the linear Kalman filter.

The rationale is that of exploiting maximum likelihood estimations in order to compute the most probable position based on the 
radio measurements (Patwari et al., 2001, Patwari et al., 2003). The maximum likelihood approach is compared with other positioning approaches based on radio measurements in terms of both accuracy and computational burden.

Depending on the number of radio transmitters used for positioning (and available at certain instant at the UAV receiver) the radio based position estimation can be more or less reliable. Then, Kalman filter is used in order to exploit vehicle dynamics to improve the estimation of its position.

Properly avoid collisions during the flight is of fundamental importance in practical applications, in particular when flying over urban areas. Similarly to (Luo et al., 2013), the goal of the approach considered here is to provide a simple strategy for collision avoidance: simplicity (and consequently requiring a low computational effort) plays a key role in order to make it possible to be run in real time in each UAV. Nevertheless, the strategy proposed in this paper significantly reduces the volume of the region forbidden for the manoeuvres of the UAVs at each time instant with respect to (Luo et al., 2013), while dealing even with the multiple device collision case (e.g. more than two UAVs involved in the possible collision).

It is worth to notice that the simplicity requirement on the collision avoidance strategy allows us to consider possible collision only in a very short future time interval. Longer intervals can be useful in order to obtain less restrictive trajectory changes, however at the cost of a significant increase in the computational complexity of the algorithm. When the operating conditions allow to consider a centralized approach, then strategies based on considering possible collisions in longer time intervals and model predictive control (MPC) can be considered (Boivin et al., 2008, Wang et al., 2007).

\section{SYSTEM DESCRIPTION}

Despite most of the positioning strategies for UAVs are typically based on the use of GPS signal, other approaches have previously considered proposed in the literature in order to reduce the positioning error (Coppa et al., 2009), or to obtain positioning when GPS signal is not available. In particular, a positioning approach based on the RSS of the WiFi signal has been considered in (Luo et al., 2013). Despite the use of RSS typically does not allow to obtain very high accurate positioning, it has the main advantage of not requiring (possibly expensive) specific ranging devices other than the WiFi receiver.

Hence, similarly to (Luo et al., 2013), this work investigates the use of WiFi RSS for positioning of UAVs. The approach presented here can be considered as evolution of that in (Luo et al., 2013) in order to improve the performance of the system.

The system is assumed to be composed by $n$ UAVs (e.g. Fig. 1) and $m \mathrm{WiFi}$ stations typically positioned on the ground. The position of the WiFi stations is assumed to be known with an accuracy higher than that expected for the positioning of the UAVs.

Each UAV is assumed to pursue a task, independently of the others. In spite of their independent tasks, communications between UAVs are allowed in order to avoid collisions: hence this aim is achieved in a cooperative fashion. Consequently, UAVs are assumed to be provided of a computational unit and of a WiFi communication device, which will be exploited in order to enhance cooperation between different vehicles.

The adopted strategy is assumed to be decentralized, i.e. each UAV uses its computational unit to modify in real time its trajectory according to collision avoidance needs and to limit the

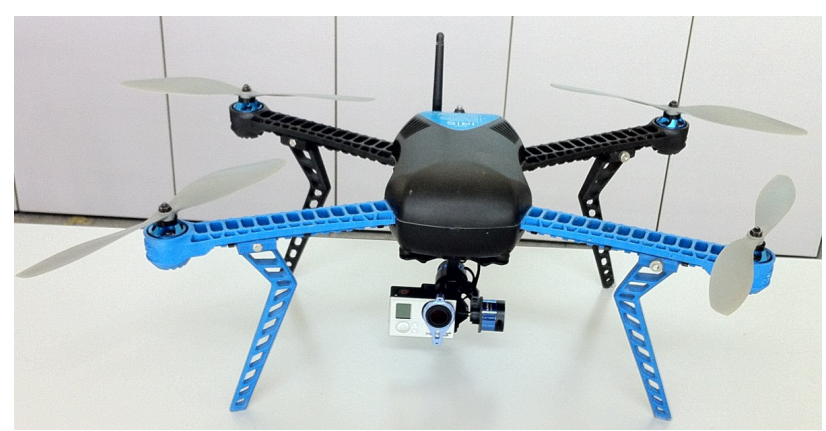

Figure 1: Example of UAV: IRIS 3D Robotics quadricopter.

error with respect to its reference trajectory (that is assumed to be known during the flight, the design of the reference trajectory is out of the scope of this paper). More specifically, all the UAVs are assumed to run the same algorithm, but with different reference trajectories, which are determined by their specific aims. Since the computational power available in mobile devices is quite limited, then the algorithm used by the each UAV for positioning and collision avoidance (e.g. to properly adapt their trajectories in order to avoid collisions with other UAVs) has to be sufficiently simple (i.e. with low computational requirements).

Each UAV is assumed to be identifiable by a unique integer number, that is associated to the priority of the task pursued by such UAV: the higher the identity number, the higher the priority of the task pursued by the UAV. When modifications of the UAV trajectories are necessary to avoid collisions, priority numbers will be used to reduce the trajectory changes (with respect to their reference trajectories) of UAVs with higher priority.

Furthermore, all the devices involved in the system are assumed to be synchronized: the synchronization process is assumed to have been already performed at the time of flight. This can be achieved quite easily by synchronizing all the device clocks to one used as reference if delays due to signal propagation can be assumed to be negligible.

\section{PRELIMINARIES}

Ranging by means of WiFi RSS is obtained by taking into account of the following model of RSS measurements (Patwari et al., 2003):

$$
P=P_{R 0}+\gamma \log \frac{d_{0}}{d}+e
$$

where $P$ is the measured signal strength (in decibel), $P_{R 0}$ is the received signal strength at a reference distance $d_{0}, d$ is the real distance between WiFi emitter and receiver, $\gamma$ is the signal attenuation coefficient (that depends on the specific environment, in outdoor applications (mostly in free space) it is (approximately) 20 ), and $e$ is the measurement noise. The above equation can be easily re-arranged as follows:

$$
P=P_{0}-\gamma \log d+e
$$

where $P_{0}$ is obtained combining $P_{R 0}$ and $\log d_{0}$.

Fig. 2 shows the results of a set of RSS measurements obtained by means of smartphones used as WiFi receivers (the specific values of the RSS can change depending on the considered devices, 
however even with different devices the results will be similar to those illustrated here with respect to the ranging goal, as described in the following). The figure shows the median RSS values at each considered distance (x-marks), whereas error-bars are used in order to show the whole range of measured RSS values. Similar results have been previously reported in the literature by other authors as well (Whitehouse et al., 2007, Stoep, 2009).

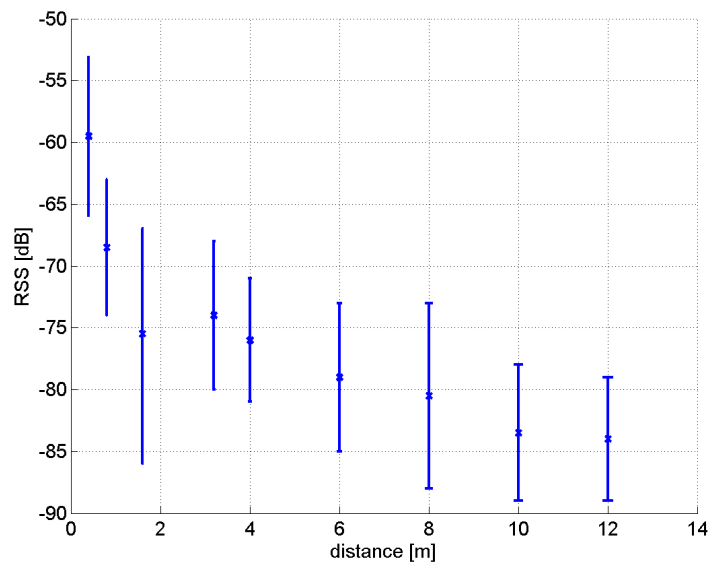

Figure 2: RSS measurements

Accordingly to the results shown in Fig. 2 and in (Whitehouse et al., 2007, Stoep, 2009), the strength of the error $d$ can vary depending on several factors. Nevertheless, in most of the cases it can be sufficiently well approximated by means of a strength value constant over all the values of $d$. Motivated by the above considerations, hereafter the RSS noise is assumed to be modeled as follows: $e \sim \mathcal{N}\left(0, \sigma^{2}\right)$.

Once an RSS measurement $P$ is available, the corresponding distance can be computed from (2):

$$
d=\exp \left(\left(P_{0}-P+e\right) / \gamma\right),
$$

where, since the value of $e$ in real applications is unknown the estimated distance is computed as follows:

$$
\hat{d}=\exp \left(\left(P_{0}-P\right) / \gamma\right)
$$

Since the dependence of $d$ from $e$ is nonlinear, it is clear that the effect of noise changes depending on the considered distance. More specifically, the same error value $e$ leads to a much larger distance estimation error for small values of the measured RSS (large values of the real distance) than when dealing with large RSS values (small values of the real distance). Then, rewriting (4) explicitly showing the distance error $e_{d}$, it follows that $\hat{d}=d+$ $e_{d}$, where the statistical characteristics of $e_{d}$ (its mean strength) depends on the real distance $d$.

The strength of the error of the positioning system is clearly strongly related with that of ranging error $e_{d}$ : consequently, a desirable working condition is to use a sufficient number of WiFi stations in order to have that in each spatial point of interest at least $\bar{m}$ of them have ranging error $e_{d}$ smaller than the desired positioning error.

It is worth to notice that at least four ranging measurements are needed in order to solve the positioning problem in a three dimensional Euclidian space. Hence $\bar{m}$ is expected to be larger or equal then four. Nevertheless, as shown in Section 4., the proposed approach allows to partially compensate missing measurements by exploiting the estimated dynamic of the vehicle.

\section{POSITIONING APPROACH}

In this work the estimation of the positions of the UAVs is obtained by means of a statistical filtering (i.e. linear Kalman filter approach). Let the UAVs be synchronized, and let $T$ be the period of the position estimation updates. At the beginning of each time period $T$ the UAVs broadcast information on their state (current and expected future position). Similarly, at each time period the UAVs evaluate the RSS values of the WiFi signals from the WiFi ground stations and from the other UAVs. Then, each UAV updates its own estimated position by taking into account of both the RSS measurements from WiFi stations and from the other UAVs in their neighborhood.

In order to simplify the notation, let $T=1 \mathrm{~s}$ in the following (it is worth to notice that $T$ can be set to smaller values in practical applications). Hence, hereafter without loss of generalization $T$ will be omitted from the equations (e.g. $(t+1)$ will be used instead of $(t+1) T)$. Then, the temporal dynamic of each UAV is modeled by means of the following dynamic model (random walk model for the velocity):

$$
\left[\begin{array}{l}
x(t+1) \\
v(t+1)
\end{array}\right]=A\left[\begin{array}{l}
x(t) \\
v(t)
\end{array}\right]+\left[\begin{array}{l}
w_{x}(t) \\
w_{v}(t)
\end{array}\right]
$$

where $x(t)$ is the spatial position of the vehicle at time step $t, v(t)$ is its velocity vector, and the matrix $A$ (modeling the temporal dynamic of the system) is defined as follows

$$
A=\left[\begin{array}{ll}
I & T \\
0 & I
\end{array}\right]
$$

and $\left[\begin{array}{ll}w_{x} & w_{v}\end{array}\right]^{\top}$ are assumed to be zero-mean Gaussian random noises, where $w_{x}$ and $w_{v}$ are assumed to be independent, and their variances are $\sigma_{x}^{2} I$ and $\sigma_{v}^{2} I$. Since the dynamic model to compute the position update is directly derived from the physics law of motion, then $\sigma_{x}$ is typically assumed to be quite small (values different from zero shall be considered in order to avoid problems related to the singularity of the Kalman gain along the corresponding directions (Soderstrom, 1994)).

Instead, the following equation will be assumed to model the measurement process of the position of each UAV (the same equation can be independently written for all the UAVs):

$$
y(t)=\left[\begin{array}{ll}
I & 0
\end{array}\right] x(t)+w_{y}(t),
$$

where $w_{y}(t)$ is the measurement noise at time $t$. More details on the measurement process will be provided in the following.

Using (5) and (7), the position estimation problem can be solved by means of a linear Kalman filter, as usual.

\subsection{Measurement process: least squares}

Collecting the measurements from all the stations, then the estimation of a UAV position $y$ at time $t$ can be formulated as the following optimization problem:

$$
y=\arg \min _{y}\left(\sum_{k}\left(\left\|q_{k}-y\right\|-\hat{d}_{k}\right)^{2}\right)
$$


where $q_{k}$ is the position of the $k$-th station, and the distance $\hat{d}_{k}$ of the UAV with respect to the $k$-th station is obtained by means of (4).

Actually, since (8) is a nonlinear problem and the system has to work in real time (i.e. it has to be as fast as possible), then an approximated (linear) version of problem (8) can be considered in order to make it simpler to solve (Li et al., 2005, Bertinato et al., 2008). In the case of perfect measurements, (8) is equivalent to the following set of equations:

$$
\begin{aligned}
\left\|q_{1}-y\right\|^{2} & =\hat{d}_{1}^{2} \\
\left\|q_{2}-y\right\|^{2} & =\hat{d}_{2}^{2} \\
& \vdots \\
\left\|q_{m}-y\right\|^{2} & =\hat{d}_{m}^{2}
\end{aligned}
$$

Then, by subtracting (for instance) from the first equation all the other ones (Bertinato et al., 2008) (or by subtracting from the above equations their average ( $\mathrm{Li}$ et al., 2005)) it is possible to formulate the problem as a linear equation (that can be solved (in least squares sense) by using the Moore-Penrose pseudo-inverse, as usual):

$$
\hat{A} y=b \quad \Rightarrow \quad y=\hat{A}^{\dagger} b
$$

where the matrices $\hat{A}$ and $b$ can be easily computed following the above considerations ( $\mathrm{Li}$ et al., 2005, Bertinato et al., 2008), and $\hat{A}^{\dagger}$ is the Moore-Penrose pseudo-inverse of $A$.

Notice that the solution of (12) is not exactly equivalent to that of (8), however in non-singular systems the two solutions are typical quite close to each other.

\subsection{Measurement process: maximum likelihood}

The maximum likelihood estimator for the positioning problem described above can be easily expressed as follows (Bertinato et al., 2008, Patwari et al., 2001, Patwari et al., 2003):

$$
y=\arg \min _{y} \sum_{k}\left(\log \frac{\left\|q_{k}-y\right\|^{2}}{\hat{d}_{k}^{2}}\right)^{2}
$$

Since the maximum likelihood estimator (13) is nonlinear its minimum has to be computed iteratively. The UAV position predicted with (5) or the linear least squares estimation can be used to initialize the iterative algorithm to compute the solution of (13). In this paper a Gauss-Newton like algorithm has been implemented in order to compute the solution of (13).

Thanks to the asymptotic efficiency of the maximum likelihood method, the variance of the estimator (13) can be approximated (i.e. lower bounded) by means of the Cramér-Rao lower bound (and the Fisher information matrix). Unfortunately, this lower bound can be quite optimistic when dealing with a finite (not so large) number of measurements. Instead, bootstrap can be used in order to overcome with issue, enabling the computation of the error covariance for the measurement $y$ to be used in the Kalman filter (5), (7). Notice that to speed up the computation only few samples shall be considered, hence the estimated covariance will be a rough estimation of the real one (it is also possible to magnify it by means of a scaling factor in order to reduce the risk of underestimating the uncertainty of the estimated position).

\subsection{Dealing with missing measurements}

In real applications, especially when using a not so large number of stations $m$, it can occasionally occur that UAV $i$ receive RSS measurements from $m_{i}$ stations, where $m_{i}<4$. When these conditions occur the 3D position of the UAV cannot be uniquely computed by using only the RSS measurements. Missing the RSS position measurement $y(t)$, its position can be inferred by using the Kalman filter prediction $\hat{x}(t \mid t-1)$, provided by taking into account of the temporal dynamic expressed in (5). However, the information provided by the $m_{i}$ measurements can still be useful to improve the prediction $\hat{x}(t \mid t-1)$. The UAV position can be computed by using a maximum likelihood approach (similar to that already considered above), but combining the $m_{i}$ measurements with the Kalman prediction $\hat{x}(t \mid t-1)$ with its uncertainty (that is assumed to be normally distributed).

\section{COLLISION AVOIDANCE}

The problem of collision avoidance has been previously considered in the literature. In particular, (Wang et al., 2007, Boivin et al., 2008) proposed approaches based on model predictive control. Despite these approaches allow to effectively deal with the collision problem, they assume the use of a cooperation strategy in a centralized fashion, that can be inefficient in certain cases, e.g. when dealing with a large number of vehicles.

Instead, (Luo et al., 2013) proposed a distributed approach, where each UAV determines its own future trajectory based on its measurements and on the information sent by the vehicles in its spatial neighborhood. Interestingly, (Luo et al., 2013) proposed the use of a very simple rule to be followed in order to avoid collisions. The use of a simple collision avoidance algorithm makes it possible to execute it directly on the computational unit of the vehicle. Furthermore, the approach in (Luo et al., 2013) considers the use of direct distance measurements between different UAVs, based on RSS ranging.

In this Section, first the so called "orthogonal rule" (for collision avoidance) proposed in (Luo et al., 2013) is summarized. Then, it is properly modified in order to make a more efficient use of the available space.

The orthogonal rule in (Luo et al., 2013) makes use of a spherical safety zone centered in the current estimated position of the vehicle. Let the considered vehicle be the $i$-th one, then the safety zone for vehicle $i$ is computed for all the others UAVs (i.e. $\forall j \neq i$ ). For each $j \neq i$ the ray $S_{i j}$ of the safety zone of vehicle $i$ is defined taking into account of the imperfect distance information of RSS ranging techniques. When vehicle $j$ is at a measured distance lower than $S_{i j}$ from $i$, then both UAVs $i$ and $j$ run the orthogonal rule algorithm (that will be described in the following) in order to avoid collision, otherwise the trajectories of the two UAVs will not be modified.

Let $\delta$ be a threshold for the RSS ranging error and let $P(\delta)$ be the probability that the ranging error is lower or equal than $\delta$. Assume that the considered algorithm for collision avoidance works properly (i.e. it allows to change the UAV trajectories in such a way to avoid collision) when the risk of collision is correctly detected. Then, one can reduce the risk of collisions by repeating $n_{d}$ independent measurements of the distance between two UAVs: the collision probability will be lower or equal to the probability that in all the $n_{d}$ measurements the error is greater than $\delta$, i.e. $(1-P(\delta))^{n_{d}}$. These considerations can be used to compute the number of measurements $n_{d}$ to be used in order to upper bound the collision probability to a specific value. For instance, if $\delta=4 \mathrm{~m}, P(\delta)=0.8$, then $n_{d}=8$ to upper bound the collision probability to $10^{-5}$.

Motivated by the above considerations, in (Luo et al., 2013) the ray $S$ has been defined as follows: 


$$
S=\left(\frac{\delta}{P(\delta)}+\left\|v_{i j} t_{r}\right\|\right) n_{d}
$$

where the first additional term considers uncertainty in the ranging measurement, while the second additional term takes into account of expected position change of the two vehicles due to their velocities $\left(v_{i j}\right.$ is the maximum relative velocity between the two vehicles). $t_{r}$ is defined as the time necessary to run the orthogonal rule algorithm and to apply the consequent trajectory changes, if necessary.

The orthogonal rule is actually a simple procedure to avoid collisions between two UAVs: let a potential collision be detected and let $j$ be the UAV with lowest priority, then the trajectory of $j$ is changed in such a way that:

- at the next considered time instant $t+1$ it is on the edge of the safety zone (at time $t$ ) of $i$,

- the direction from $x_{j}(t)$ to $x_{j}(t+1)$ is orthogonal to $v_{i}(t)$ (subscript indexes indicate the UAV to which variables are referred to),

- among all the possible spatial positions which satisfy the above conditions it is chosen the one that minimize the distance from $x_{j}(t)$.

Despite the above approach has the advantage of being very simple, it can have certain issues:

- it typically leads to very large safety zones, restricting the UAV manoeuvering space probably more then necessary. For instance, with $\left|v_{i j}\right|=10 \mathrm{~m} / \mathrm{s}, t_{r}=1 \mathrm{~s}, n_{d}=8$ as in (Luo et al., 2013), then $S=120 \mathrm{~m}$.

- as shown in the previous section, the error statistical characteristics change with the value of the real distance, hence the considerations on the measurement error probability are partially inconsistent (to improve the performance of the system one should consider the error distribution as a function of the measurement value, this however makes the whole approach much more complex).

- despite repeating the distance measurement between two UAV allow to reduce the error probability, it can lead to a significant delay (since measurements have to be independent, they have to be separated by at least the channel coherence time (e.g. 25ms)).

- the orthogonal rule is defined for a potential collision between two UAVs. Even if this is clearly the most obvious potential collision case, in certain applications more UAVs can be involved in a more articulated potential collision, hence the collision avoidance strategy shall be formulated in a more general case.

Taking into account of the above considerations, a different collision avoidance approach is presented in the following.

The sampling period used in this section in the collision avoidance algorithm can be set to a different value with respect to the $T$ used in the positioning algorithm (e.g. it can be a multiple of $T)$. Nevertheless, the notation will be similar to that used in the previous section, $T$ will be omitted from equations and time $t$ will be used instead of $t T$.
Uncertainty in the position $x_{i}(t)$ of the $i$-th UAV is expressed as a covariance matrix $\Sigma_{i}(t)$ as shown in Section 4.. Three principal directions can be extracted from the covariance matrix, where typically the strength of the positioning error along each of such directions is different: $\sigma_{1}^{2}, \sigma_{2}^{2}, \sigma_{3}^{2}$. In order to simplify the collision avoidance algorithm the strength of the error (e.g. its variance) along each of such directions is set to the maximum value (i.e. $\bar{\sigma}^{2}=\max _{i}\left(\sigma_{i}^{2}\right)$ ). Let $n_{d}$ be such that the probability of having a positioning error larger than $n_{d} \bar{\sigma}$ is very small (e.g. $\left.n_{d}=4\right)$

Let $\bar{x}_{i}(t+1)$ be the expected position for the UAV at time $t+$ 1 according to the trajectory designed to achieve its task. The expected UAV future position at time $t+1$ (up to the algorithm knowledge until time $t)$ is assumed to be $\bar{x}_{i}(t+1)$. If the time interval between $t$ and $t+1$ is sufficiently small, and the UAV control system appropriately works, then the uncertainty on the UAV position $\bar{x}_{i}(t+1)$ is quite similar to that of $x_{i}(t)$, hence the probability of having an error larger than $\bar{\sigma}$ shall be small.

Then, in this case the safety zone for UAV $i$ is defined as the cylinder with hemispherical ends obtained by linking the two spheres corresponding to $\bar{\sigma}$ errors at time $t$ and $t+1$, as shown in Fig. 3 .

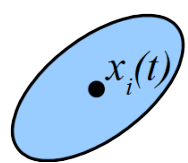

(a)

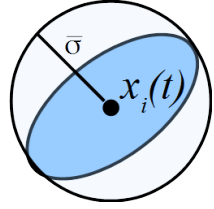

(b)

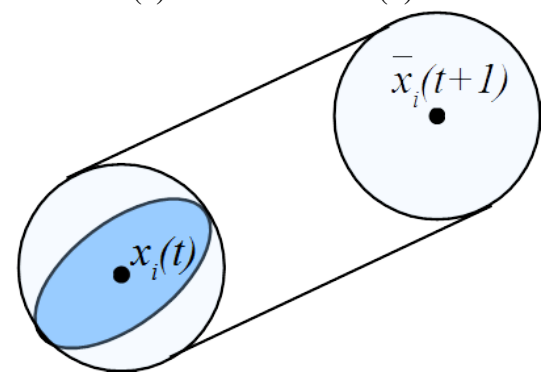

(c)

Figure 3: UAV safety zone, shown in 2D to ease the readability of the figure. (a) uncertainty on the $x_{i}(t)$ position. (b) spherical upper bound of the uncertainty on the $x_{i}(t)$ position. (c) UAV safety zone between position at time $t$ and expected position at time $t+1$.

For each UAV a safety zone (similar to Fig. 3) can be computed as described above. Each UAV sends to its neighbors the characteristics of its current safety zone, its estimated position and its priority number.

Then a potential collision is detected if there is a nonempty intersection between the safety zones of UAV $i$ and $j$, for certain values of $i$ and $j$ with $i \neq j$. Despite using spherical safety zones as in (Luo et al., 2013) the detection of potential collisions is extremely easy, the use of cylinders with hemispherical ends can allow to reduce the size of the UAV safety zones (hence increasing the UAV manoeuvering space) while making the detection of potential collision only few more complex.

All the UAVs run the same collision detection and avoidance algorithm. Hence, assuming that all the UAVs involved in a potential collision receive the same information from each other (if they are involved in a potential collision, they are supposed to be quite close to each other, and hence it is quite realistic to assume 
the absence of communication packet loss). Once a potential collision is detected (by all the UAVs involved in the potential collision), then the UAV trajectories are modified as described in the following.

Let $n_{c}$ be the number of UAVs involved in the potential collision, then assume that enough space is available along the vertical direction (free of obstacles) in order to distribute the UAVs at different altitudes. The increasing order of altitudes will be distributed according to the current order of UAV altitudes (e.g. the UAV that is currently at the highest altitude will be at the highest altitude even after the trajectory modifications): if two (or more) UAVs are at the same altitude their identification number is used in order to decide how to modify their altitudes. The minimum altitude will be the minimum admissible one according to the a priori information on the environment available to the UAVs.

\section{SIMULATION RESULTS}

The results of this section has been obtained by means of the simulation on Matlab of a system with a system with $7 \mathrm{WiFi}$ ground stations (positioned as the red x-marks in Fig. 5), and random UAV trajectories simulated in order to ensure quite smooth movements (blue line in Fig. 5 shows an example of simulated trajectory). The area of interest (because of the presence of WiFi stations or because close to the UAV trajectory) is assumed to be relatively small: in accordance with this assumption the whole system is represented in a 3D Euclidian space, whose coordinate system is $(u, v, z)$, where the space spanned by $(u, v, 0)$ is the (ground) horizontal plane. The noise on the RSS measurements is assumed to be gaussian, with standard deviation $2 \mathrm{~m}$ independent of the distance.

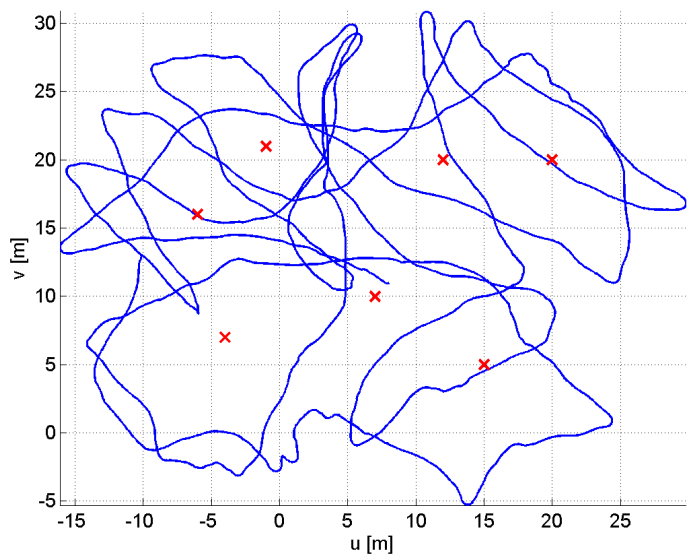

Figure 4: Positions of WiFi stations (red x-marks) on the $(u, v)$ plane, and example of simulated UAV trajectory (blue line).

According with our simulations of the above described system on more than 10000 time samples, the root mean square (RMS) positioning error obtained by means of the linear least squares method (12) is approximately $20 \mathrm{~m}$, the RMS error obtained by means of likelihood measurements (13) is $5.8 \mathrm{~m}$, whereas that obtained by means of the combination of maximum likelihood measurements and Kalman filtering (13),(5),(7) is $4.3 \mathrm{~m}$. Hence, the linear least squares method performs much worse than the other methods, and combining Kalman filtering with ML measurements allows to improve the estimates obtained by using only the ML measurements of $26 \%$, approximately. Fig. 5 shows a comparison of maximum likelihood and Kalman filter positioning errors in a time interval of 120 time samples.
It is worth to notice that the numerical results reported above are strictly related to the specific characteristics of the considered system: changing the values of certain parameter can change the numerical results. Nevertheless, the considerations derived above hold for a wide range of system conditions: maximum likelihood estimations are typically much better than linear least squares ones, and exploiting the temporal dynamic of the system allows to obtain a further estimation improvement.

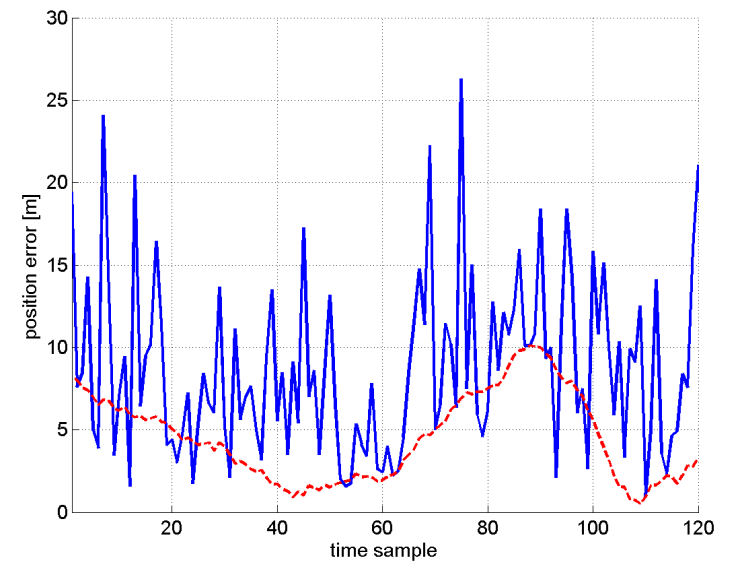

Figure 5: Positioning error: maximum likelihood (blue line) and Kalman (red dashed line) estimations.

Despite the RMS error obtained combining ML measurements and Kalman filtering is approximately $4.3 \mathrm{~m}$, Fig. 5 shows that maximum error values can be significantly larger than their average value. Accordingly with this observation and with the considerations made in the previous section, the ray of the spherical boundary in Fig. 3(b) is set to $n_{d} \bar{\sigma}$, where $n_{d}=4$, while $\bar{\sigma}$ is set accordingly to the current UAV position uncertainty. For instance, let $\bar{\sigma}=4.5 \mathrm{~m}$ and let the velocity of the vehicle be $10 \mathrm{~m}$ per time period, approximately. Then, the size of the cylinder with hemispherical ends (Fig. 3(c)) along its principal axis is given by $2 n_{d} \bar{\sigma}$ added to the vehicle velocity, i.e. $46 \mathrm{~m}$. The size of cylinder along the other two directions is equal to the diameter of each of the two spheres in Fig. 3(c) is $36 \mathrm{~m}$. Hence, despite the simulation conditions are quite similar to those previously considered for (Luo et al., 2013), the cylinder with hemispherical ends (Fig. 3(c)) defines a clearly smaller safety zone. Nevertheless, in hundreds of simulations the adopted strategy allows to properly detect potential collisions, and, consequently, avoid them.

Since the average positioning error obtained in the simulations presented in this section, $4 \mathrm{~m}$ approximately, can be too large for certain applications, different operating conditions and further improvements on the positioning algorithm can be necessary. It is worth to notice that if the UAV can fly at quite high altitudes, increasing the density of ground WiFi stations may not lead to significant improvements in the positioning error. Instead, better results can be obtained in environments where the measurement noise has a lower strength (i.e. when the UAV flight area is quite large and mostly free).

Further improvements and experimental validations of the method will be considered in our future investigations.

\section{CONCLUSIONS}

This paper considers the problem of positioning UAVs by means of WiFi RSS measurements. Different positioning methods have 
been compared: according to the considered simulations the combination of maximum likelihood positioning estimations and Kalman filtering allow to obtain definitely much lower positioning errors with respect to linear least squares and maximum likelihood estimators.

The approach proposed for collision avoidance allows to reduce the size of the safety zone with respect to the case presented in (Luo et al., 2013), thus increasing the usable space for the UAVs, while ensuring good performance. Furthermore, if there is enough free space for the UAVs to freely change their trajectories, the considered strategy allows to deal with the case of multiple UAVs potential collisions. Instead, when the trajectories of the UAVs are limited by other constraints, a more complex strategy shall be considered. This will be object of our future investigation.

\section{REFERENCES}

Bertinato, M., Ortolan, G., Maran, F., Marcon, R., Marcassa, A., Zanella, F., Zambotto, M., Schenato, L. and Cenedese, A., 2008. $\mathrm{Rf}$ localization and tracking of mobile nodes in wireless sensors networks: Architectures, algorithms and experiments.

Boivin, E., Desbiens, A. and Gagnon, E., 2008. Uav collision avoidance using cooperative predictive control. In: Control and Automation, 2008 16th Mediterranean Conference on, pp. 682688 .

Casbeer, D. W., Kingston, D. B., Beard, R. W. and McLain, T. W., 2006. Cooperative forest fire surveillance using a team of small unmanned air vehicles. International Journal of Systems Science 37(6), pp. 351-360.

Coppa, U., Guarnieri, A., Pirotti, F. and Vettore, A., 2009. Accuracy enhancement of unmanned helicopter positioning with lowcost system. Applied Geomatics 1(3), pp. 85-95.

Guarnieri, A., Vettore, A. and Camarda, M., 2010. A low cost rc-heli UAV for mapping applications. ISPRS - International Archives of the Photogrammetry, Remote Sensing and Spatial Information Sciences.
Li, Z., Trappe, W., Zhang, Y. and Nath, B., 2005. Robust statistical methods for securing wireless localization in sensor networks. In: Proceedings of the 4th international symposium on Information processing in sensor networks, IEEE Press, p. 12.

Luo, C., McClean, S. I., Parr, G., Teacy, L. and De Nardi, R., 2013. Uav position estimation and collision avoidance using the extended kalman filter. Vehicular Technology, IEEE Transactions on 62(6), pp. 2749-2762.

Maza, I., Caballero, F., Capitán, J., Martínez-de Dios, J. and Ollero, A., 2011. Experimental results in multi-uav coordination for disaster management and civil security applications. Journal of intelligent \& robotic systems 61(1-4), pp. 563-585.

Patwari, N., Dea, R. and Wang, Y., 2001. Relative location in wireless networks. In: Vehicular Technology Conference, 2001. VTC 2001 Spring. IEEE VTS 53rd, Vol. 2, IEEE, pp. 1149-1153.

Patwari, N., Hero III, A. O., Perkins, M., Correal, N. S. and O'dea, R. J., 2003. Relative location estimation in wireless sensor networks. Signal Processing, IEEE Transactions on 51(8), pp. 2137-2148.

Soderstrom, T., 1994. Discrete-time stochastic systems. Springer.

Stoep, J. V., 2009. Design and Implementation of Reliable Localization Algorithms using Received Signal Strength, MSc Thesis. University of Washington.

Wang, X., Yadav, V. and Balakrishnan, S., 2007. Cooperative uav formation flying with obstacle/collision avoidance. Control Systems Technology, IEEE Transactions on 15(4), pp. 672-679.

Whitehouse, K., Karlof, C. and Culler, D., 2007. A practical evaluation of radio signal strength for ranging-based localization. ACM SIGMOBILE Mobile Computing and Communications Review 11(1), pp. 41-52.

Zhang, C. and Kovacs, J. M., 2012. The application of small unmanned aerial systems for precision agriculture: a review. Precision agriculture 13(6), pp. 693-712. 